nanoparticles of organic fluorescent semiconductor materials. The research team said that these techniques have previously been applied to isolated, single-polymer chains (molecules) of conjugated polymers. The investigation of single morphological domains of nanoparticles of organic dyes are of particular interest, as these affect their fluorescent properties, which may find wide application in promising devices such as organic light-emitting diodes (OLEDs). The researchers reported the characterization of $N, N^{\prime}$-bis(2,5-di-tertbutylphenyl)-3,4,9,10-perylenedicarboximide (DBPI) nanoparticles by atomic force microscopy (AFM), bulk optical spectroscopy, and single-molecule/particle optical spectroscopy.

The researchers said that DBPI nanoparticles were prepared using the reprecipitation method. The optical emission spectra of the nanoparticles showed a mixture of monomer-like (blue, $538 \mathrm{~nm}$ and $578 \mathrm{~nm}$ ) and excimer-like (red, $637 \mathrm{~nm}$ ) emission. The nanoparticle size and shape were characterized by tapping-mode AFM. They said that a broad size distribution of platelike nanoparticles was observed, varying in height from 1-13 nm and in width from 10-45 nm. All the samples were mixtures of blue and red nanoparticles. The researchers were able to separate blue nanoparticles from red nanoparticles by centrifugation. The density and size of the red particles was such that the centrifugation process removed red particles from fractions with lower sucrose gradients, leaving only blue particles. However, the researchers could not obtain a fraction that contained only red particles.

According to the researchers, the two types of particles in the samples probably possess different crystal morphology, designated $\alpha$ and $\beta$ forms. The $\alpha$-perylene has a dimeric crystal structure with red emission spectra, and $\beta$-perylene has a monomeric crystal structure with blue emission spectra. Alternatively, the morphological difference could find its origin in the size of the nanoparticles, said the researchers; small amorphous particles could show blue emission and the larger crystalline particles could show red emission.

Three types of fluorescence time trajectories were found for the nanoparticles: trajectories with constant intensity, trajectories with intensity fluctuations, and trajectories that showed blinking. Time transients of red particles showed only constant intensity or intensity fluctuations. Blue particles, however, showed all three types of behavior. Blinking is caused by reversible oxidation/reduction events at special sites in the polymer backbone to which the excitation energy is efficiently funneled, said the researchers. DBPI nanoparticles could develop low-energy trap sites, which may result in fluorescence blinking. Intensity fluctuations, however, are a typical observation for multichromophoric systems in which the chromophores are not coupled. The researchers said that a significant fraction of the nanoparticles (25\%) exhibited fluorescence intermittence (i.e., "blinking"). According to the researchers, blinking in a multichromophoric system, that is, an ensemble of chromophores-in this case, nanoparticles of polymers-is evidence of efficient interchromophoric coupling and energy transfer.

VIVEK RANJAN

\section{Structure Affects Optical Limiting in Aqueous Suspensions of CNTs}

Materials used for optical limiting (OL) exhibit nonlinear optical effects including 2-photon reverse saturable absorption and nonlinear scattering. The latter has been demonstrated with suspensions of carbon black and of single-walled carbon nanotubes (SWNTs). The OL mechanism for SWNTs involves the formation and growth of bubbles that scatter incident radiation. However, a better understanding of the effect of microstructure is required before efficient OL devices can be fabricated from SWNTs. Toward this end, N. Izard from Centre Technique d'Arcueil and Université Montpellier II, France; P. Billaud and D. Riehl from Centre Technique d'Arcueil; and E. Anglaret from Université Montpellier II have recently measured the effects on OL of the length and diameter of nanoobjects composed of carbon nanotubes.

As reported in the June 15 issue of Optics Letters (p. 1509), Izard and co-researchers demonstrated that tubular nano-objects with larger diameters $(d)$ display lower OL thresholds and better efficiencies than those with smaller diameters. The researchers prepared aqueous suspensions from commercially available SWNTs and multiwalled carbon nanotubes (MWNTs) at concentrations of $\sim 10 \mathrm{mg} / \mathrm{L}$, yielding a linear optical transmission at $532 \mathrm{~nm}$ of $\sim 70 \%$ for 2 -mm-thick cells. SWNT samples included either bundles of nanotubes $(d=10-15 \mathrm{~nm})$ or individual nanotubes $(d=1.4 \mathrm{~nm})$. The SWNT bundles contained either long $(>1 \mu \mathrm{m})$ or shortened $(<100 \mathrm{~nm})$ nanotubes. MWNT samples had a fivefold variation in length but only about a twofold variation in $d(10-20 \mathrm{~nm}$ or 20-50 nm). The researchers performed nonlinear transmittance measurements using a $15 \mathrm{~ns}$ optical pulse at a $532 \mathrm{~nm}$ optical wavelength. They also performed optical pump-probe measurements using a
$532 \mathrm{~nm}$ pump pulse with a duration of $4 \mathrm{~ns}$ and a continuous probe beam at $633 \mathrm{~nm}$. The researchers based their conclusions on three observations of transmittance spectra: (1) comparable OL for all MWNT suspensions; (2) nearly identical OL for SWNT bundles with the same $d$; and (3) OL thresholds of $100 \mathrm{~mJ} / \mathrm{cm}^{2}, 200 \mathrm{~mJ} / \mathrm{cm}^{2}$, and $400 \mathrm{~mJ} / \mathrm{cm}^{2}$ for MWNTs $(d=20-50 \mathrm{~nm})$, SWNT bundles, and individual SWNTs, respectively.

Interpreting the results of their pumpprobe experiments, the researchers attributed the diameter effect to differences in bubble nucleation and growth: Individual SWNTs nucleated smaller bubbles than SWNT bundles, which, following Laplace's law, requires more energy. Conversely, bundled SWNTs nucleated larger bubbles at lower incident fluences. Furthermore, individual SWNTs exhibited poorer OL efficiency than bundles, because smaller bubbles take longer to reach a critical size. Finally, although this interpretation is consistent with their results, the researchers said that they cannot rule out effects that involve faster heating of nanotubes in bundles than individual nanotubes. Optical absorption in carbon nanotubes is primarily due to plasmons, which are sensitive to the environment (including nanotube bundling). The researchers said that their results are also consistent with plasmon coupling between CNTs in bundles, which may induce substantial absorption enhancement.

STEVEN TROHALAKI

\section{Catalytic Asymmetric Procedure Enables Synthesis of Apple Leafminer Pheromones from Isoprenoid Building Blocks}

The saturated isoprenoid unit is an essential building block for vitamins, pheromones, chlorophyll, marine natural products, and archaebacterial lipids, so its synthesis attracts the interest of researchers in the field of total synthesis. Most of the methods, such as chiral pool strategies, enzymatic desymmetrization protocols, and chiral auxiliary-based approaches, are multistep reactions. They usually need to start with a stoichiometric amount of chiral material, and they cannot deliver all diastereoisomers. In order to improve this synthesis, the groups of R. Noyori (Nagoya University) and E. Negishi (Purdue University) did some initial studies on asymmetric catalysis synthesis and provided some of the desired compounds. However, A. Minnaard from the Stratingh Institute at the University of Groningen, the Netherlands, reported in Chemical Communications (p. 1387; DOI: 10.1039/b419268k), 